\title{
Respiratory syncytial virus induces functional thymic stromal lymphopoietin receptor in airway epithelial cells
}

This article was published in the following Dove Press journal:

Journal of Inflammation Research

22 March 2013

Number of times this article has been viewed

\author{
Michael M Miazgowicz ${ }^{1,2}$ \\ Molly S Elliott ${ }^{3}$ \\ Jason S Debley ${ }^{3}$ \\ Steven F Ziegler ${ }^{1,2}$
}

'Immunology Program, Benaroya Research Institute, Seattle, WA,

${ }^{2}$ Department of Immunology,

University of Washington School of

Medicine, Seattle, WA,${ }^{3}$ The Center

for Immunology and Immunotherapies,

Seattle Children's Research Institute,

Seattle, WA, USA
Correspondence: Steven F Ziegler Benaroya Research Institute, I20 I Ninth Ave, Seattle, WA 9810I, USA

Tel +l 2062875657

Fax +I 2063426572

Email sziegler@benaroyaresearch.org

\begin{abstract}
The epithelial-derived cytokine thymic stromal lymphopoietin (TSLP) plays a key role in the development and progression of atopic disease and has notably been shown to directly promote the allergic inflammatory responses that characterize asthma. Current models suggest that TSLP is produced by epithelial cells in response to inflammatory stimuli and acts primarily upon dendritic cells to effect a $\mathrm{T}$ helper type 2-type inflammatory response. Recent reports, however, have shown that epithelial cells themselves are capable of expressing the TSLP receptor (TSLPR), and may thus directly contribute to a TSLP-dependent response. We report here that beyond simply expressing the receptor, epithelial cells are capable of dynamically regulating TSLPR in response to the same inflammatory cues that drive the production of TSLP, and that epithelial cells produce chemokine $\mathrm{C}-\mathrm{C}$ motif ligand 17 , a T helper type 2-associated chemokine, in response to stimulation with TSLP. These data suggest that a direct autocrine or paracrine response to TSLP by epithelial cells may initiate the initial waves of chemotaxis during an allergic inflammatory response. Intriguingly, we find that the regulation of TSLPR, unlike TSLP, is independent of nuclear factor kappa-light-chain-enhancer of activated B cells, suggesting that the cell may be able to independently regulate TSLP and TSLPR levels in order to properly modulate its response to TSLP. Finally, we show evidence for this dynamic regulation occurring following the viral infection of primary epithelial cells from asthmatic patients. Taken together, the data suggest that induction of TSLPR and a direct response to TSLP by epithelial cells may play a novel role in the development of allergic inflammation.
\end{abstract}

Keywords: TSLP, TSLPR, RSV, asthma, epithelium

\section{Introduction}

Asthma is a chronic inflammatory disease of the airways, classically associated with strong $\mathrm{T}$ helper type 2 (Th2)-type immune responses, including airway hyperresponsiveness, eosinophilic cell infiltration, goblet cell metaplasia, and mucus hypersecretion. ${ }^{1}$ Although the etiology of asthma is complex, it is now appreciated that the epithelial-derived cytokine thymic stromal lymphopoietin (TSLP) plays a key role in mediating various aspects of the disease. In mouse models, overexpression of TSLP, or exogenous administration via the airways, is sufficient to drive the development of all the classical hallmarks of asthmatic disease. ${ }^{2,3}$ TSLP is furthermore required for the development of allergic asthma; mice lacking the TSLP receptor (TSLPR) do not develop eosinophilic airway inflammation following challenge with experimental allergen. ${ }^{2,4}$ TSLP appears to act both to initiate and to sustain an inflammatory response against foreign antigens when it acts at the appropriate stages of that response. 
In humans, high levels of TSLP are believed to drive asthmatic responses in a similar fashion. Elevated levels of TSLP are present in the lungs of asthmatic patients, ${ }^{5}$ and the induced expression of TSLP has been linked to several known risk factors for the development of asthma. Of particular note, severe childhood infections with respiratory syncytial virus (RSV) have long been known to positively correlate with the subsequent development of childhood asthma. ${ }^{6}$ Interestingly, recent studies have shown that RSV is capable of inducing high levels of TSLP production in airway epithelial cells. ${ }^{7}$ Intriguingly, this induction is enhanced in airway epithelial cells from asthmatic individuals, implying that the inappropriate sensitivity of these cells to inflammatory stimuli may be linked to a TSLP-driven asthmatic response. ${ }^{7}$

Although much attention has recently naturally been focused on TSLP and its role in allergic disease, less is known about the regulation and expression of its receptor. Recent reports have shown that TSLPR is expressed on cells of both the airway and colonic epithelium, where it has been shown to play a role in the wound-healing response. ${ }^{8,9} \mathrm{We}$ show here that TSLPR is inducibly expressed on the cells of the airway epithelium. Furthermore, we present the novel observation that this induction is driven by the same stimuli that drive TSLP production, albeit by a parallel mechanism, and show that this induced receptor is capable of functionally responding to TSLP. Finally, we show that much as airway epithelial cells displayed an enhanced induction of TSLP, airway epithelial cells from asthmatic individuals also have an enhanced induction of TSLPR in response to infection by RSV. We speculate that this enhanced induction, in addition to allowing the epithelial cells to directly mediate a TSLP-dependent inflammatory response, may be symptomatic of an underlying sensitivity that had originally predisposed these individuals to asthma, and that by upregulating both the cytokine and its receptor, epithelial cells can establish an autocrine/paracrine feedback loop that may serve to enhance and sustain the TSLP-dependent inflammatory response.

\section{Materials and methods Reagents and constructs}

Recombinant human tumor necrosis factor- $\alpha$ (TNF- $\alpha$ ) and phycoerythrin (PE)-conjugated murine $\alpha$-human TSLPR (clone 1A6) were purchased from eBioscience (San Diego, CA, USA). Recombinant human TSLP was kindly provided by Dr Michael Comeau (Amgen, Seattle, WA, USA). BAY11-7082 was purchased from EMD Millipore (Billerica,
MD, USA). Full-length TSLPR and interleukin-7 receptor- $\alpha$ (IL-7R $\alpha$ ), lacking a stop codon, were amplified from cDNA obtained from TNF- $\alpha$ treated A549 cells, and cloned into a mammalian expression vector using the pcDNA3.1/V5-His TOPO TA Expression Kit from Invitrogen (Carlsbad, CA, USA). Expression vectors containing full-length nuclear factor kappa-light-chain-enhancer of activated B cells (NF- $\mathrm{KB})$ p65 or p50 subunits were created as previously described. ${ }^{10}$ The -404 CIS promoter-luciferase (cis-luc) and purified RSV (pRSV)- $\beta$-galactosidase reporter (pRSV- $\beta$-gal) were described previously. ${ }^{10,11}$

\section{Submerged cell culture and viral infection}

The human lung epithelial cell line A549 was obtained from the American Type Culture Collection (Manassas, VA, USA), maintained in Dulbecco's modified Eagle medium, and supplemented with $10 \%$ fetal bovine serum, $1 \%$ penicillin/ streptomycin, nonessential amino acids, and sodium pyruvate. Normal human bronchial epithelial cells (NHBECs) were obtained from Lonza (Walkersville, MD, USA) and maintained according to the manufacturer's instructions. RSV (line 19, A strain) was obtained and propagated as previously described. ${ }^{7}$ Virus was added to A549 or NHBECs at the titers indicated. Cells were washed and received fresh media after 2 hours, then harvested for analysis 24 hours following the initial infection.

\section{Air-liquid interface culture and viral infection}

Primary bronchial epithelial cells (BECs) were obtained from healthy and asthmatic children, cultured under air-liquid interface (ALI) conditions, and infected with RSV as previously described. ${ }^{7}$ Briefly, BECs were collected by unsheathed cytologic brushings performed via an endotracheal tube during elective surgical procedures. Cells at passage 2 or 3 were subsequently seeded onto transwells and grown under ALI conditions. BECs were exposed at the apical surface to RSV line 19 at multiplicity of infection of 0.5 or an equivalent volume of fluid from the control Vero cell supernatant for 2 hours, and cells collected for analysis 96 hours postinfection. At study enrolment, the disease severity of each asthmatic patient was classified according to National Institutes of Health (NIH) Guidelines for the Diagnosis and Management of Asthma (ref: NIH guidelines: http://www.nhlbi.nih.gov/ guidelines/asthma/index.htm), and history of acute exacerbations of asthma was assessed through questionnaire and review of medical records. The protocol was approved by the Seattle Children's Hospital Institutional Review Board. 


\section{Real-time quantitative polymerase chain reaction analysis}

Total RNA and cDNA preparation was performed as previously described. ${ }^{10}$ Human primers used were purchased from Sigma-Aldrich (St Louis, MO, USA), and include TSLPR (forward, 5'-CAGAGCAGCGAGACGACATT-3'; reverse, 5'-GGTACTGAACCTCATAGAGG-3'), IL-7R $\alpha$ (forward, 5'-GCTCAGGGGAGATGGATCCT-3'; reverse, 5'-GTCTTCTTATGATCGGGGAG-3'), and hypoxanthineguanine phosphoribosyltransferase (HPRT; forward, 5'-TGGCGTCGTGATTAGTGATG-3'; reverse 5'-GCACACAGAGGGCTACAATG-3'). Primer sequences for TSLP and chemokine $\mathrm{C}-\mathrm{C}$ motif ligand 17 (CCL17) were previously published. ${ }^{12,13}$

\section{Western blots}

Samples were lysed with $1 \times$ lysis buffer (50 mM Tris-8.0, $150 \mathrm{mM} \mathrm{NaCl}, 1.0 \%$ Triton $\mathrm{X}-100$ ), and total protein concentration determined using the Bio-Rad Protein Assay (BioRad, Hercules, CA, USA), according to the manufacturer's protocol. Equal amounts of protein were electrophoresed on NuPAGE 10\% Bis-Tris gels (Invitrogen) and transferred onto membranes. TSLPR was detected with murine $\alpha$-human TSLPR clone 1A6, and membranes stripped and reprobed for glyceraldehyde 3-phosphate dehydrogenase (GAPDH) and detected via rabbit $\alpha$-human GAPDH (Trevigen, Gaithersburg, MD, USA).

\section{Transfections}

Prior to transfection, cells were seeded in wells and allowed to reach a confluency between $50 \%$ and $70 \%$. Plasmid constructs were mixed with Mirus TransIT-LT1 transfection reagent (Mirus, Madison, WI, USA) according to the manufacturer's protocol, and applied to cell cultures. All transfections were allowed to rest at 12-16 hours prior to further experimental manipulation. For the signal transducer activator of transcription 5 (STAT5) luciferase assays, expression vectors containing TSLPR and IL-7R $\alpha$, the cis-luc promoter-reporter construct, and pRSV- $\beta$-gal control plasmid were transfected into cells at a ratio of 1:1:2:1, respectively. For transfections of TSLPR and IL-7R $\alpha$ expression vectors alone, constructs were added in equal amounts.

\section{Luciferase assays}

A549 cells were transfected and rested as described. Cells were subsequently stimulated for 8 hours with recombinant TSLP, washed with phosphate buffered saline, and immediately lysed in $200 \mu \mathrm{L}$ lysis buffer
(Promega, Madison, WI, USA). Lysates were analyzed for luciferase activity using Luciferase Assay Reagent (Promega), and read using a Lumat LB9507 luminometer (Berthold, Bad Wildbad, Germany). $\beta$-galactosidase levels were detected using the $\beta$-galactosidase Enzyme Assay System (Promega), following the manufacturer's protocol.

\section{Statistical analysis}

All experiments were analyzed for statistical significance. Where samples were compared with an internal control and reported as a "fold change," statistical significance was calculated with a one-sample $t$-test using the null hypothesis that the population mean is equal to 1 . In all other cases, two samples were compared using Student's $t$-test or other appropriate statistical tests as indicated. Statistical significance is indicated on the figures, where $P=0.05$ is used as the minimum threshold for significance.

\section{Results}

TNF- $\alpha$ induces TSLPR and IL-7R $\alpha$ expression in human airway epithelial cells TSLPR is a heterodimer consisting of the cytokine-specific TSLPR chain and the IL-7R $\alpha$ chain that it shares with the IL-7 receptor. Although it is well established that the receptor is expressed on cells of hematopoietic origin, recent reports have suggested that both TSLPR and IL-7R $\alpha$ are expressed by epithelial cells in both the lungs and gut., Although we have previously shown that proinflammatory stimuli can induce the production of TSLP by lung epithelial cells, ${ }^{7,10}$ it is not known whether TSLPR can be modulated under similar conditions. To assess whether the TSLPR can be induced under inflammatory conditions, we stimulated the human airway epithelial cell line A549 cells with the cytokine TNF- $\alpha$. Upregulation of TSLPR mRNA was detectable by 4 hours, rising to a steady increase of four-fold over unstimulated cells by 8 hours (Figure 1A). Likewise, TNF- $\alpha$ induced IL-7R $\alpha$ mRNA as early as 1 hour (data not shown), reaching a peak of eight-fold upregulation over unstimulated cells by 4 hours, and dropping off to a two-fold upregulation at 24 hours poststimulation (Figure 1A). These results demonstrate that TSLPR and IL-7R $\alpha$, like TSLP, are subject to dynamic regulation under inflammatory conditions.

\section{Infection of epithelial cells with RSV potently induces both TSLPR and IL-7R $\alpha$}

$\mathrm{RSV}$ is a common respiratory virus and a known risk factor for the development of asthma. ${ }^{6,14} \mathrm{We}$ have previously shown that RSV infection potently upregulates TSLP in 

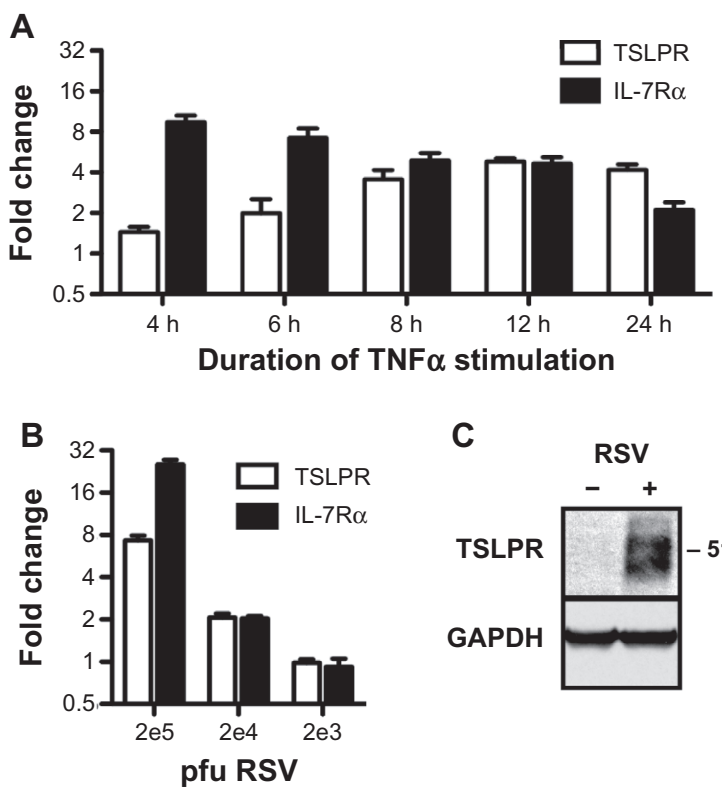

C
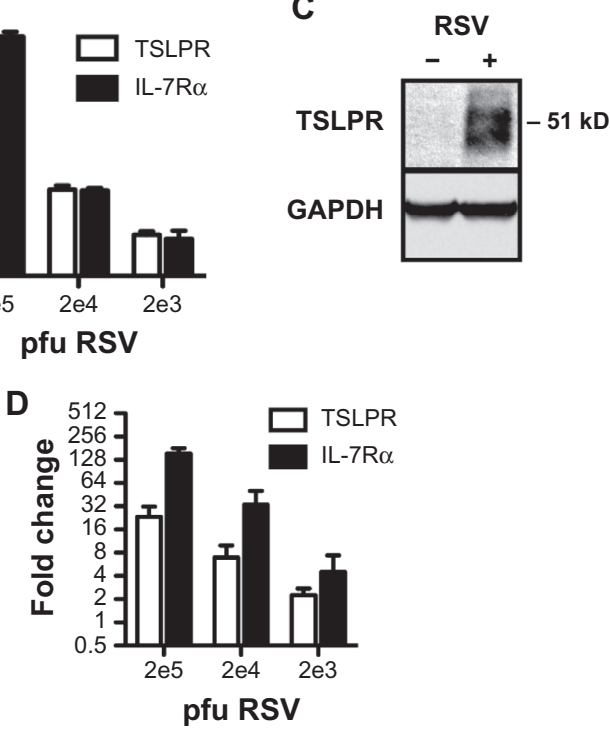

Figure I Induction of thymic stromal lymphopoietin receptor (TSLPR) and interleukin-7 receptor- $\alpha$ (IL-7R $\alpha$ ) in epithelial cells. (A) A549 cells were treated with $40 \mathrm{ng} / \mathrm{mL}$ tumor necrosis factor- $\alpha$ (TNF- $\alpha$ ) for the indicated times and subjected to quantitative polymerase chain reaction ( $\mathrm{QPCR}$ ) analysis. Values are reported as a fold change over untreated cells. (B) A549 cells were infected with the indicated titers of respiratory syncytial virus (RSV) for 2 hours and harvested 24 hours postinfection for qPCR analysis. Values are reported as a fold change over mock-infected cells. (C) Representative Western blots of TSLPR and glyceraldehyde 3-phosphate dehydrogenase (GAPDH) from A549 cells mock-infected or infected with $2 \times 10^{5}$ pfu RSV. (D) Normal human bronchial epithelial cells (NHBECs) were infected with the indicated titers of RSV for 2 hours and harvested 24 hours postinfection for $\mathrm{qPCR}$ analysis.

Notes: Values are reported as a fold change over mock-infected cells. All qPCR data are reported as the mean \pm standard error of the mean of at least three independent experiments. All mRNA levels are normalized to hypoxanthine-guanine phosphoribosyltransferase (HPRT).

both primary human airway epithelial cells and epithelial cell lines. ${ }^{7}$ To determine whether RSV infection could also induce TSLPR and IL-7R $\alpha$, we infected A549 cells with various titers of RSV for 2 hours, and assessed mRNA levels 24 hours postinfection. Compared with mock-infected cells, at titers of $2 \times 10^{4}$ and $2 \times 10^{5} \mathrm{pfu}$, RSV induced a two-fold and seven-fold upregulation, respectively, of TSLPR, and a two-fold and 25-fold upregulation, respectively, of IL-7R $\alpha$ (Figure 1B). TSLPR protein was readily detectable at these levels (Figure 1C).

These effects were even more pronounced in primary epithelial cells; NHBECs were similarly infected with different titers of RSV and showed strong upregulation of both TSLPR and IL-7R $\alpha$. Induction of TSLPR was detectable at as low as $2 \times 10^{3}$ pfu RSV, upregulating levels by two-fold over mock-infected cells, and increasing to up to 23 -fold upregulation at $2 \times 10^{5} \mathrm{pfu}$. Likewise, IL-7R $\alpha$ mRNA was upregulated between four-fold and 150-fold, respectively, at the same range of titers (Figure 1D). Taken together, these results demonstrate that TSLPR and IL-7R $\alpha$ can be and are induced by the same inflammatory stimuli that drive TSLP production in airway epithelial cells.

\section{Regulation of TSLPR and IL-7R $\alpha$ is not mediated by NF- $\kappa B$}

TNF- $\alpha$ and RSV, although distinctly different stimuli, utilize similar signaling pathway intermediates; both are capable of signaling through canonical NF- $\mathrm{KB}$ as well as p38-kinase, c-Jun N-terminal kinase (JNK), and extracellular signalregulated kinase (ERK) pathways. ${ }^{15,16}$ Given that previous studies from our laboratory have shown that TSLP expression is dependent on the activity of NF- $\kappa \mathrm{B},{ }^{10}$ it was reasonable to infer that NF- $\kappa$ B also regulates TSLPR and IL-7R $\alpha$ expression. To test whether this is the case, we transfected A549 cells with expression vectors expressing either the p50 or p65 subunits of $\mathrm{NF}-\kappa \mathrm{B}$, and assayed for gene expression 24 hours following transfection. Surprisingly, although we observed the previously reported p65-dependent TSLP induction, ${ }^{10}$ neither TSLPR nor IL-7R $\alpha$ were upregulated by either p50 or p65 (Figure 2A). To confirm these results, we pretreated A549 cells with the I $\kappa \mathrm{B} \alpha$ inhibitor BAY-11-7082, followed by treatment with TNF- $\alpha$,and assayed 4 hours later for suppression of TSLP, TSLPR, and IL-7R $\alpha$ induction. Consistent with our previous results, the induction of TSLP was blocked in the presence of the inhibitor, and TSLPR and IL-7R $\alpha$ levels were indistinguishable from cells treated with TNF- $\alpha$ and vehicle alone (Figure 2B). In a similar fashion, the inhibitor failed to affect the upregulation of TSLPR or IL-7R $\alpha$ following RSV infection (data not shown). We conclude from these data that in contrast to TSLP, TSLPR and IL-7R $\alpha$ are not dependent on canonical NF- $\kappa$ B.

\section{Epithelial cells expressing TSLPR can functionally respond to TSLP}

Much of the previous research on TSLP has focused on its effects on hematopoietic cells, where it has been shown to drive the maturation of both Th2 CD4+ T cells and dendritic cells, and stimulate the production of chemokines such as CCL17. ${ }^{2,5}$ Less well studied are the effects of TSLP on epithelial cells. Although previous studies have suggested a 

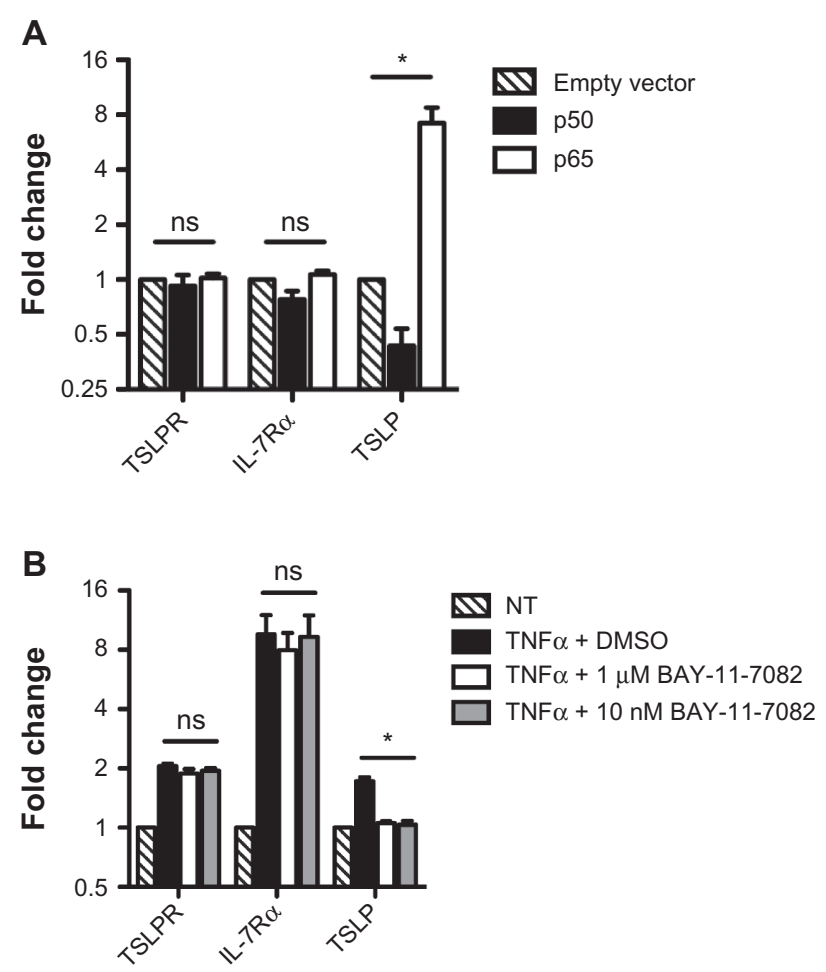

Figure 2 Thymic stromal lymphopoietin receptor (TSLPR) and interleukin-7 receptor- $\alpha($ IL-7R $\alpha)$ are not regulated by nuclear factor kappa-light-chain-enhancer of activated $B$ cells (NF- $\mathrm{KB}$ ). (A) A549 cells were transfected with empty vector or

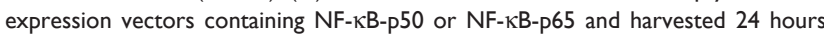
post-transfection for quantitative polymerase chain reaction analysis. (B) A549 were pretreated with dimethyl sulfoxide (DMSO), I $\mu$ M BAY-I I-7082, or $10 \mathrm{~nm}$ BAY-I I7082 for 30 minutes, then treated with $40 \mathrm{ng} / \mathrm{mL}$ tumor necrosis factor- $\alpha$ (TNF- $\alpha$ ) for 4 hours and harvested for quantitative polymerase chain reaction analysis. Notes: All data are reported as the mean \pm standard error of the mean of at least three independent experiments. mRNA levels are normalized to hypoxanthineguanine phosphoribosyltransferase (HPRT) and expressed relative to empty vector or untreated control samples, respectively. ${ }^{*} P<0.05$; ns indicates no significant difference.

role for TSLP in assisting with an IL-13-mediated woundhealing response in airway epithelial cells, ${ }^{8}$ we wanted to assess whether epithelial cells could be directly involved with a TSLP-mediated Th2-type response.

Our data suggest that by upregulating both the cytokine and its receptor, epithelial cells may be able to respond to TSLP in an autocrine or paracrine fashion. As identifying TSLP-driven functional responses in epithelial cells could be complicated by these potential autocrine/paracrine effects, we created expression vectors expressing TSLPR and IL-7R $\alpha$ in order to force expression of the full TSLPR complex while bypassing the need for stimulation that may upregulate native TSLP expression. We transfected both subunits into A549 cells and stimulated the transfected cells with recombinant TSLP in order to determine whether epithelial cells are capable of responding to TSLP.

Previous studies have shown that TSLP is an activator of STAT5. ${ }^{17-19}$ To determine whether TSLP activates
STAT5 in epithelial cells, we made use of a reporter construct containing luciferase under the control of an STAT5-responsive element from the human cytokineinducible SH2-containing protein promoter. ${ }^{11}$ We transfected A549 cells with the reporter in combination with our TSLPR and IL-7R $\alpha$ expression constructs, and treated the cells for 8 hours with varying concentrations of recombinant TSLP. Compared with untreated cells, TSLP treatment induced a 1.5-fold upregulation of STAT5-dependent luciferase activity at all concentrations of TSLP tested (Figure 3A). Although modest, this effect was statistically significant, and is consistent with similarly modest TSLP-dependent responses reported elsewhere. ${ }^{8}$

CCL17 is a known TSLP-target gene and plays a central role in recruiting many of the cell types associated with the canonical TSLP-dependent inflammatory response. ${ }^{20} \mathrm{As}$ it is known that epithelial cells can express CCL17, ${ }^{21}$ we tested whether TSLP can drive CCL17 expression in TSLPR+ epithelial cells. We transfected A549 cells with TSLPR and IL-7R $\alpha$ expression constructs and enriched for TSLPR+ cells using an $\alpha$-TSLPR antibody. These enriched TSLPR+ A549 cells were then treated with $50 \mathrm{ng} / \mathrm{mL}$ of recombinant TSLP for 8 hours. We found that TSLP treatment upregulated CCL17 levels more than two-fold compared with similarly enriched but untreated cells. Taken together, these results indicate that epithelial cells can indeed respond to TSLP, and that they can do so by participating in known Th2-associated response patterns.

\section{Asthmatic BECs upregulate TSLPR and IL-7R $\alpha$ following RSV infection}

Although these data strongly indicate that airway epithelial cells are capable of upregulating a functional TSLPR complex in response to common inflammatory stimuli, we wanted to assess whether epithelial cells behaved the same way in a more physiological setting. In order to more closely mimic the microenvironmental conditions airway epithelium is naturally exposed to, we collected primary BECs from bronchial brushings of both healthy and asthmatic individuals, and cultured the cells under ALI conditions. ${ }^{7}$

We first assessed what the levels of TSLPR and IL-7R $\alpha$ expression were under basal conditions in both healthy and asthmatic individuals. Expressed as a percentage of the levels of the housekeeping gene HPRT, we found no significant difference between the mean expression of TSLPR in healthy versus asthmatic samples (Figure 4A). The overall level of TSLPR expression was comparable with the levels observed in submerged NHBEC cultures (data not shown). In contrast, 
A

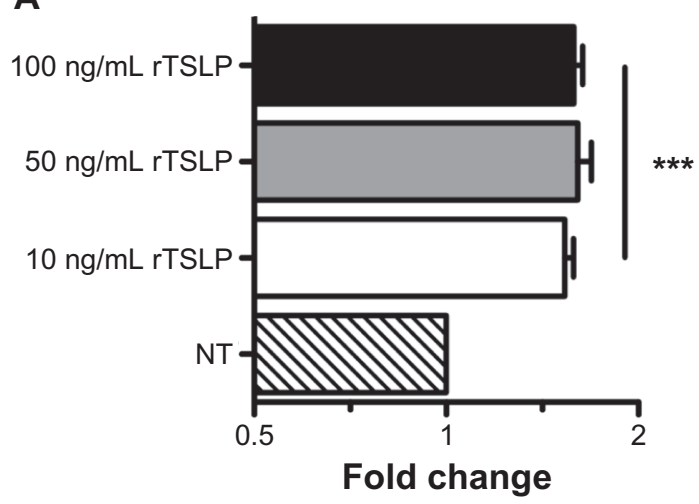

B

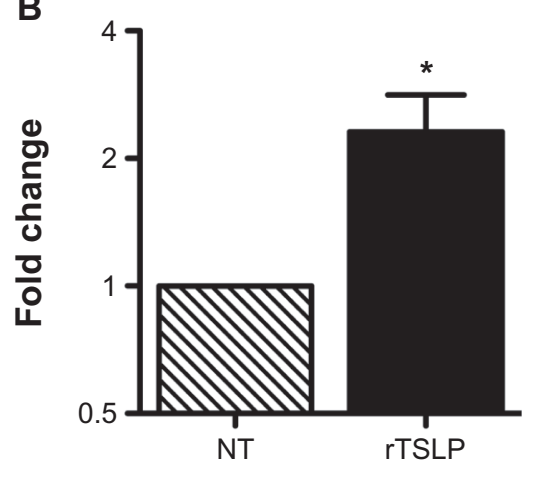

Figure 3 Epithelial cells functionally respond to thymic stromal lymphopoietin (TSLP). (A) A549 cells were cotransfected with TSLP receptor (TSLPR) and interleukin-7 receptor- $\alpha(I L-7 R \alpha)$ expression constructs along with the cis-luc reporter construct and the pRSV- $\beta$-gal control vector. Following transfection, cells were stimulated with indicated amounts of recombinant TSLP for 8 hours, harvested, and assayed for luciferase activity. Activity levels were normalized to $\beta$-gal levels, and expressed relative to untreated cells (***P $<0.00$ I vs untreated cells). (B) A549 cells were cotransfected with TSLPR and IL-7R $\alpha$ expression constructs.

Notes: Following transfection, TSLPR+ cells were enriched with an $\alpha$-TSLPR antibody and treated with $50 \mathrm{ng} / \mathrm{mL}$ recombinant TSLP. Cells were harvested after 8 hours and analyzed via quantitative polymerase chain reaction for expression of CCLI7, normalized to hypoxanthine-guanine phosphoribosyltransferase (HPRT), and expressed relative to untreated cells ( $* P=0.031$ vs untreated cells). All data are reported as the mean \pm standard error of the mean of at least six independent experiments.

we found that asthmatic samples did express significantly lower levels of IL-7R $\alpha$ compared with healthy samples (Figure 4A), and overall levels from both healthy and asthmatic groups were between three and five times lower than IL-7R $\alpha$ levels observed in submerged NHBEC cultures.

We next assessed whether BECs could upregulate TSLPR and IL-7R $\alpha$ in response to RSV infection. Matching samples from each patient analyzed were apically exposed to RSV and harvested 4 days later. Expressed now as a fold change relative to each sample's respective basal expression, we found that asthmatic samples, but not healthy samples, significantly upregulated TSLPR levels over baseline. TSLPR levels were upregulated by an average of 2.6-fold, with a $95 \%$ confidence interval of \pm 1.1 in the asthmatic group, in contrast to 1.5 -fold \pm 0.6 in the healthy group (Figure 4B). These data mirror previous data showing that TSLP levels are increased only following RSV infection in samples from asthmatic individuals. ${ }^{7}$

With regard to IL-7R $\alpha$, samples displayed a more heterogeneous response to RSV, with some samples upregulating IL-7R $\alpha$ as much as 50 -fold, and others displaying no change in levels at all. Overall, both healthy and asthmatic samples displayed a trend toward upregulating IL-7R $\alpha$ but failed to meet the criteria for statistical significance with $P$-values of 0.086 and 0.059 , respectively (Figure 4B). It is worthwhile to note, however, that the raw expression values of IL-7R $\alpha$ in infected samples (as a percentage of HPRT) were not significantly different between healthy and asthmatic groups, in contrast to the differences seen under basal conditions.
We interpret this to indicate that IL-7R $\alpha$ levels are truly upregulated, at least in the asthmatic group, and that the statistical significance is simply being masked by high variance between samples.

Asthma is a progressive disease with a diverse array of symptoms, and the severity of the disease can vary widely between individual patients or even within the same patient over time. Given that TSLPR expression is controlled by inflammatory cues, we reasoned that TSLPR expression may correlate with disease severity. Asthmatic individuals were clinically categorized into mild intermittent, mild persistent, and moderate persistent classifications of disease severity based on NIH Guidelines for the Diagnosis and Management of Asthma (ref: NIH guidelines: http://www.nhlbi.nih.gov/ guidelines/asthma/index.htm). In addition, individuals were separately grouped depending on whether they had or had not been previously treated in an emergency department or hospital one or more times for acute asthma exacerbations, a marker of severe disease. There was a trend toward a positive association between TSLPR expression following RSV infection and disease classification, although the differences did not reach statistical significance (Figure 4C). In contrast, there was statistical significance $(P=0.04)$ between history of exacerbations and TSLPR expression following RSV infection (Figure 4D). These effects were seen only following infection with RSV, and thus imply an enhanced sensitivity to RSV or propensity to upregulate TSLPR that correlates with more severe manifestations of asthmatic disease. 
A

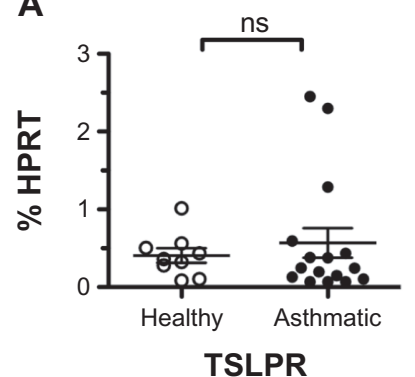

B

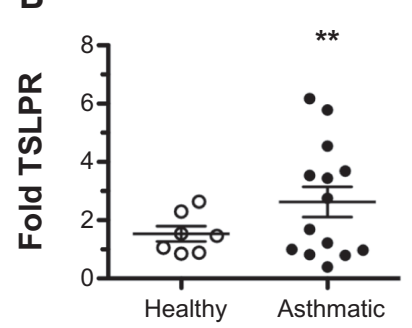

C

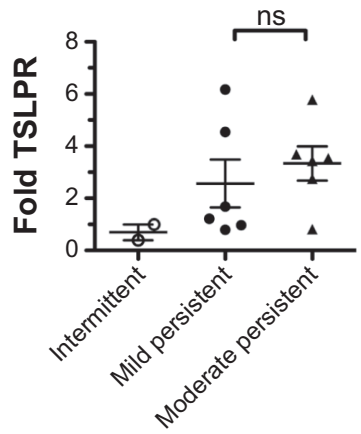

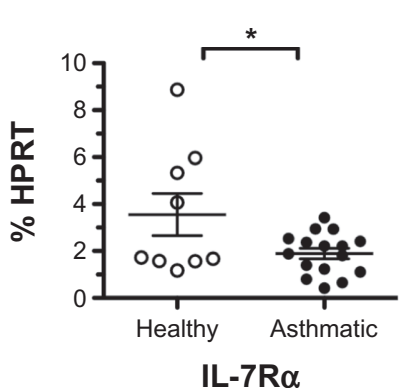

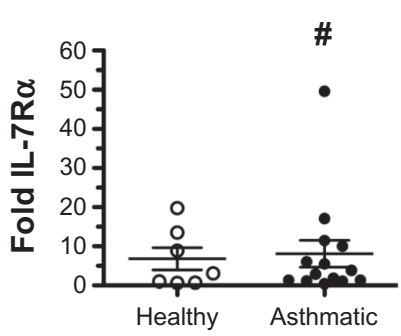

D

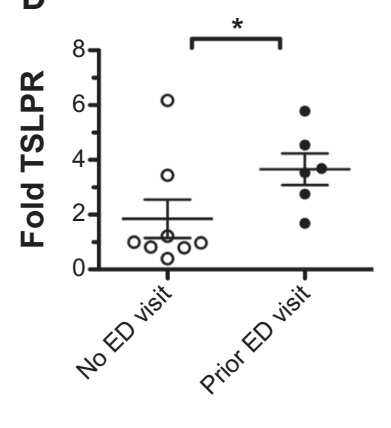

Figure 4 Primary bronchial epithelial cells from asthmatic individuals upregulate thymic stromal lymphopoietin receptor (TSLPR) following infection with respiratory syncytial virus (RSV). (A) Bronchial epithelial cells from healthy and asthmatic patients were collected via brushings, proliferated in submerged culture, then differentiated in air-liquid interface (ALI) culture. Basal condition ALI cultures were apically exposed to control Vero cell supernatants, and RNA was collected for quantitative polymerase chain reaction analysis. Levels of TSLPR and interleukin-7 receptor- $\alpha$ (IL-7R $\alpha$ ) are expressed as a percentage of hypoxanthine-guanine phosphoribosyltransferase (HPRT) levels from each sample. Each data point represents an individual patient, and mean values \pm standard error of the mean are reported. Healthy and asthmatic patients were grouped and compared with Student's $t$-test $(* P=0.03$; ns indicates no significant difference). (B) Matching ALI cultures from each patient were infected with RSV at a multiplicity of infection of 0.5 , and RNA collected for quantitative polymerase chain reaction analysis. Expressions of TSLPR and IL-7R $\alpha$ were normalized to HPRT and reported as a fold change over the respective basal condition sample. Each data point represents an individual patient, and mean upregulation \pm standard error of the mean are reported. Healthy and asthmatic groups were evaluated for significant upregulation at the $95 \%$ confidence interval $(* * P=0.008$; \# indicates $P=0.059)$. (C) Asthmatic individuals were clinically categorized as having mild intermittent (open circles), mild persistent (closed circles), or moderate persistent (closed triangles) disease, and samples were grouped accordingly for analysis. Levels of TSLPR induced by RSV infection, as determined previously, were grouped according to these disease classifications, and compared with Student's t-test (ns indicates no significant difference). (D) Asthmatic individuals were categorized as having (closed circles) or not having (open circles) a history of asthma exacerbations requiring emergency department (ED) care or hospitalization. Levels of TSLPR induced by RSV infection, as determined previously, were grouped according to these classifications, and compared with a Mann-Whitney $U$ test $(* P=0.04)$

\section{Discussion}

Investigations into the interplay between the regulation of TSLP by epithelial cells and the development of Th2-type inflammatory responses have provided keen insights into the etiology of atopic disease. More recently, however, it is becoming apparent that the regulation of TSLPR in epithelial cells may also play an interesting role in how atopic responses are generated. We have shown here that both TSLPR and IL-7R $\alpha$ can be upregulated in epithelial cells by both the general proinflammatory cytokine TNF- $\alpha$ and by infection with RSV. Both stimuli have previously been shown to upregulate TSLP, and, more significantly, RSV infection, in particular, is known to correlate with the development and exacerbation of asthmatic disease. ${ }^{6,14}$ Our data suggest that epithelial cells are capable of initiating an autocrine/paracrine TSLP signaling circuit, thereby directly initiating or propagating the TSLP-driven Th2-type inflammatory responses associated with RSV infection and other forms of airway inflammation. Indeed, we find that stimulation of TSLPR+ epithelial cells drives the production of the Th2 chemokine CCL17, allowing epithelial cells to directly participate in a Th2-type response.

Despite being downstream of the same kinds of stimuli, we have presented data indicating that TSLP and TSLPR expression are, in fact, regulated by different intracellular signaling pathways. Based on what is known about both TNF- $\alpha$ - and RSV-induced signaling pathways, we speculate that TSLPR and IL-7R $\alpha$ may be regulated by a p38-kinase-, JNK-, or ERK-mediated pathway. Nevertheless, although the precise pathway controlling TSLPR and IL-7R $\alpha$ is still unknown, we have ruled out the NF- $\mathrm{KB}$ pathway as a contributor to their expression. That NF- $\kappa B$ is the central regulator of TSLP expression ${ }^{10}$ creates an interesting situation where epithelial cells have the ability to selectively modulate their own responsiveness to TSLP without otherwise interrupting TSLP-dependent responses in other cells. Indeed, although we have looked at only two representative stimuli in this study - TNF- $\alpha$ and RSV there may be other more selective stimuli that drive the expression of only TSLPR and IL-7R $\alpha$ without affecting TSLP or vice versa. By upregulating only the receptor, the epithelium may be able to prime itself for a rapid TSLP-driven response only after further environmental requirements have been met. Conversely, by upregulating only TSLP, the epithelium can delay such a response until after the responding cells have integrated the proper environmental cues. 
We can see evidence for this type of regulation occurring when we examine primary BECs from healthy and asthmatic patients under basal conditions. Previous research has found that asthmatic epithelium produces significantly greater levels of TSLP, ${ }^{7}$ whereas our data indicate that levels of TSLPR are equivalent between healthy and asthmatic cells. Furthermore, we find that IL-7R $\alpha$ levels are lower in asthmatic cells. Given that IL-7R $\alpha$ is known in other cell types to undergo downregulation upon stimulation, ${ }^{22,23}$ we believe that, taken together, our data indicate that epithelial cells from asthmatic individuals have previously responded to an inflammatory stimulus that was sufficient to elevate their production of TSLP but insufficient to sustain expression of TSLPR. As we know that epithelial cells are capable of directly participating in a Th2-type response upon TSLP stimulation, expressing lower levels of the receptor may allow epithelium to limit the perpetuation of this response in the face of chronic inflammation.

Under basal conditions, our data suggest that expression of the TSLPR complex is roughly equivalent between healthy and asthmatic individuals, or at the very least subject to subtle regulatory compensations. In contrast, we have demonstrated that epithelial cells from asthmatic individuals have a significantly higher propensity to upregulate TSLPR compared with their healthy counterparts, and that this effect particularly correlates with a history of exacerbations indicative of severe forms of the disease. Taken together with our data showing that signaling through TSLPR can lead to epithelial production of CCL17, we describe a system in which epithelial cells from more severely asthmatic individuals can express higher levels of TSLPR, leading to enhanced TSLPdependent production of proasthmatic factors, in turn enhancing the severity of the disease. Whether this mechanism is truly a positive feedback loop - that enhanced asthmatic inflammation leads to enhanced expression of TSLPR - or whether our data instead describe an underlying genetic or environmental predisposition toward enhanced TSLPR expression that is part of an overall asthmatic predisposition remains an open question.

Recent work has begun to suggest that asthma is likely a disease of the airway epithelium, with the triggering of the characteristic inflammatory responses due to dysregulation of that epithelium. ${ }^{24-26}$ Our work is consistent with this model of disease initiation. ALI cultures generated using cells from asthmatic individuals are capable of inducing both TSLP and TSLPR when exposed to respiratory viruses or proinflammatory cytokines. Whether this dysregulation represents an inherent difference between healthy and asthmatic epithelial cells or is a consequence of the chronic inflammatory state of the airways in asthmatics is unclear. There are reports of structural changes in the airway epithelium in asthmatics, ${ }^{25}$ as well as difference in epigenetic patterns between cells from healthy and asthmatic individuals. ${ }^{27}$ Together with our data, these differences suggest that airway epithelial cells from healthy and asthmatic individuals respond differently from environmental insult. Consistent with this hypothesis, we have found that the intracellular sensor for RSV, RIG-I, is induced more strongly in asthmatic airway epithelial cells following RSV infection. ${ }^{7}$ What role this plays in regulating TSLPR expression following RSV infection remains to be determined. Taken as a whole, the data presented here provide further evidence of the role of airway epithelial cells in asthma, and suggest a role for TSLP signaling in those cells.

\section{Acknowledgments}

We thank Whitney Xu and Theingi Aye for excellent technical assistance; Drs Daniel Campbell, Joan Goverman, and Daniel Bowen-Pope for critical discussion of the manuscript before submission; and members of the Ziegler laboratory for helpful discussions throughout the duration of this work. We thank Sylvia McCarty for administrative support. This work was funded in part from grants AI068731 and HL098067 to SFZ and HL102708 to SFZ and JSD.

\section{Disclosure}

The authors report no conflicts of interest in this work.

\section{References}

1. Cohn L, Elias JA, Chupp GL. Asthma: mechanisms of disease persistence and progression. Annu Rev Immunol. 2004;22:789-815.

2. Zhou B, Comeau MR, De Smedt T, et al. Thymic stromal lymphopoietin as a key initiator of allergic airway inflammation in mice. Nat Immunol. 2005;6:1047-1053.

3. Headley MB, Zhou B, Shih WX, Aye T, Comeau MR, Ziegler SF. TSLP conditions the lung immune environment for the generation of pathogenic innate and antigen-specific adaptive immune responses. J Immunol. 2009;182:1641-1647.

4. Al Shami A, Spolski R, Kelly J, Keane-Myers A, Leonard WJ. A role for TSLP in the development of inflammation in an asthma model. $J$ Exp Med. 2005;202:829-839.

5. Ying S, O'Connor B, Ratoff J, et al. Thymic stromal lymphopoietin expression is increased in asthmatic airways and correlates with expression of Th2-attracting chemokines and disease severity. J Immunol. 2005; $174: 8183-8190$.

6. Sigurs N, Aljassim F, Kjellman B, et al. Asthma and allergy patterns over 18 years after severe RSV bronchiolitis in the first year of life. Thorax. 2010;65:1045-1052.

7. Lee HC, Headley MB, Loo YM, et al. Thymic stromal lymphopoietin is induced by respiratory syncytial virus-infected airway epithelial cells and promotes a type 2 response to infection. JAllergy Clin Immunol. 2012;130: $1187-1196$. 
8. Semlali A, Jacques E, Koussih L, Gounni AS, Chakir J. Thymic stromal lymphopoietin-induced human asthmatic airway epithelial cell proliferation through an IL-13-dependent pathway. J Allergy Clin Immunol. 2010;125:844-850.

9. Reardon C, Lechmann M, Brüstle A, et al. Thymic stromal lymphopoetin-induced expression of the endogenous inhibitory enzyme SLPI mediates recovery from colonic inflammation. Immunity. 2011; 35:223-235

10. Lee HC, Ziegler SF. Inducible expression of the proallergic cytokine thymic stromal lymphopoietin in airway epithelial cells is controlled by NFkappaB. Proc Natl Acad Sci U S A. 2007;104:914-919.

11. Verdier F, Rabionet R, Gouilleux F, et al. A sequence of the CIS gene promoter interacts preferentially with two associated STAT5A dimers: a distinct biochemical difference between STAT5A and STAT5B. Mol Cell Biol. 1998;18:5852-5860.

12. Lee HC, Headley MB, Iseki M, Ikuta K, Ziegler SF. Cutting edge: inhibition of NF-kappaB-mediated TSLP expression by retinoid X receptor. Cutting Edge J Immunol. 2008;181:5189-5193.

13. Kumagai N, Fukuda K, Nishida T. Synergistic effect of TNF-alpha and IL-4 on the expression of thymus- and activation-regulated chemokine in human corneal fibroblasts. Biochem Biophys Res Commun. 2000; 279:1-5.

14. Johnston SL, Pattemore PK, Sanderson G, et al. Community study of role of viral infections in exacerbations of asthma in 9-11 year old children. BMJ. 1995;310:1225-1229.

15. Baker SJ, Reddy EP. Modulation of life and death by the TNF receptor superfamily. Oncogene. 1998;17:3261-3270.

16. Mikkelsen SS, Jensen SB, Chiliveru S, et al. RIG-I-mediated activation of p38 MAPK is essential for viral induction of interferon and activation of dendritic cells: dependence on TRAF2 and TAK1. J Biol Chem. 2009;284:10774-10782.
17. Isaksen DE, Baumann H, Zhou B, et al. Uncoupling of proliferation and Stat5 activation in thymic stromal lymphopoietin-mediated signal transduction. J Immunol. 2002;168:3288-3294.

18. Isaksen DE, Baumann H, Trobridge PA, Farr AG, Levin SD, Ziegler SF. Requirement for stat5 in thymic stromal lymphopoietin-mediated signal transduction. J Immunol. 1999;163:5971-5977.

19. Levin SD, Koelling RM, Friend SL, et al. Thymic stromal lymphopoietin: a cytokine that promotes the development of IgM+ B cells in vitro and signals via a novel mechanism. J Immunol. 1999;162:677-683.

20. Homey B, Zlotnik A. Chemokines in allergy. Curr Opin Immunol. 1999;11:626-634.

21. Sekiya T, Miyamasu M, Imanishi M, et al. Inducible expression of a Th2-type CC chemokine thymus- and activation-regulated chemokine by human bronchial epithelial cells. J Immunol. 2000;165:2205-2213.

22. Vranjkovic A, Crawley AM, Gee K, Kumar A, Angel JB. IL-7 decreases IL-7 receptor alpha (CD127) expression and induces the shedding of CD127 by human CD8+ T cells. Int Immunol. 2007;19:1329-1339.

23. Hammerbeck CD, Mescher MF. Antigen controls IL-7R alpha expression levels on $\mathrm{CD} 8 \mathrm{~T}$ cells during full activation or tolerance induction. J Immunol. 2008;180:2107-2116.

24. Holgate ST. The epithelium takes centre stage in asthma and atopic dermatitis. Trends Immunol. 2007;28:248-251.

25. Holgate ST. Epithelial dysfunction in asthma. J Allergy Clin Immunol. 2007;120:1233-1244.

26. Kato A, Schleimer RP. Beyond inflammation: airway epithelial cells are at the interface of innate and adaptive immunity. Curr Opin Immunol. 2007;19:711-720.

27. Stefanowicz D, Hackett TL, Garmaroudi FS, et al. DNA methylation profiles of airway epithelial cells and PBMCs from healthy, atopic and asthmatic children. PLoS ONE. 2012;7:e44213.
Journal of Inflammation Research

\section{Publish your work in this journal}

The Journal of Inflammation Research is an international, peer-reviewed open-access journal that welcomes laboratory and clinical findings on the molecular basis, cell biology and pharmacology of inflammation including original research, reviews, symposium reports, hypothesis formation and commentaries on: acute/chronic inflammation; mediators of inflamma-

\section{Dovepress}

tion; cellular processes; molecular mechanisms; pharmacology and novel anti-inflammatory drugs; clinical conditions involving inflammation. The manuscript management system is completely online and includes a very quick and fair peer-review system. Visit http://www.dovepress.com/ testimonials.php to read real quotes from published authors. 